\title{
Controlling the Velocity and Kinetic Energy of an Ideal Gas: An EWMA Control Chart and Its Average Run Length
}

\author{
Yupaporn AREEPONG ${ }^{1}$ and Rapin SUNTHORNWAT ${ }^{2, *}$ \\ ${ }^{I}$ Department of Applied Statistics, Faculty of Applied Science, King Mongkut's University of Technology \\ North Bangkok, Bangkok 10800, Thailand \\ ${ }^{2}$ Industrial Technology Program, Faculty of Science and Technology, Pathumwan Institute of \\ Technology, Bangkok 10330, Thailand
}

("Corresponding author's e-mail: rapin@pit.ac.th)

Received: 2 March 2020, Revised: 14 December 2020, Accepted: 12 December 2020

\begin{abstract}
An ideal gas is a gas in the form of a particle or molecule. Its velocity and kinetic energy are interesting topics in several studies in physical chemistry. This research aims to evaluate the average run length based on the exponentially weighted moving average statistic for its molecular velocity and kinetic energy of Maxwell-Boltzmann distribution. Derivation of the integral equation, which is equal to the average run length and numerical method of the integral equation, was applied to evaluate the average run length of a gas molecule's molecular velocity and kinetic energy. The Trapezoidal rule as numerical method and its error was analyzed for approximation of average run length. The findings showed that the average run length of molecular velocity decreased in the higher temperature with the given mass of the molecule. Moreover, there was a decrease in the average run length of molecular kinetic energy in the higher temperature.
\end{abstract}

Keywords: Maxwell-Boltzmann distribution, Average run length, Integral equation, Ideal gas, Molecular velocity, Kinetic energy

\section{Introduction}

An ideal gas is a gas in theory with fundamental assumptions of a small particle with random movement and the molecular collisions with no lost energy [1,2]. In physical chemistry, the kinetic molecular theory and its applications of an ideal gas are interesting topics for many researchers. The calculation of molecular fluxes and equivalent pressure in ideal gases was conducted and described by deriving mathematical expressions [3]. The ideal gas law was evaluated to sufficiently describe the consistency of the experiment and its effect on the greenhouse problem in the determination of the radiative forcing [4]. The mathematical formula for molar mas based on ideal gas law is illustrated to describe the greenhouse effect [5]. Furthermore, applying the concept of the ideal gas was created to be an algorithm to solve the optimization technique problem in engineering [6] and simulation under gravitational field [7].

In applied statistics, the control chart is mostly applied to many problems such as in the production process and economics and in terms of controlling and evaluation. In particular, one of the tools for measuring the performance of the control chart is the evaluation of the average run length. The expected value of the run length before the occurrence of the false alarm signal is popularly employed on the Exponentially Weighted Moving Average (EWMA) control chart and Cumulative Sum (CUSUM) control chart. The average run length can be classified into 2 types: average run length of in control state $\left(A R L_{0}\right)$ and average run length of out-of-control state $\left(A R L_{1}\right)$ which is $A R L_{0}$ with changed parameters. Moreover, the 
standard $A R L_{0}$ is approximately 370. There are three methods for evaluating the average run length: Monte Carlo simulation, Martingale method, and the integral equation. Integral equations and integro-differential equation can be solved by analytical and numerical evaluation method [8-12]. Several researches studied the evaluation of the average run length. For example, Martingale approach for average run length of EWMA and CUSUM control charts on autoregressive process was presented. The results showed that Martingale approach is efficient method and better than Monte Carlo simulation method [13].

The integral equation method was employed to determine the analytical and numerical average run length on the EWMA control chart and CUSUM control chart with observations followed by Laplace distribution and Hyperexponential distribution. It was found that the analytical average run length was better than the numerical average run length $[14,15]$. Analytical and numerical average run length based on the integral equation of EWMA control chart with the long memory autoregressive fractionally integrated moving average was investigated. The finding showed that the analytical average run length was better in performance than the numerical average run length [16,17]. Adaptive EWMA control chart to evaluate average run length by using a discrete-time Markov chain was designed for time-varying smoothing parameter. The optimization technique was adopted to calculate the average run length [18].

The application in modern physical chemistry involved the employment of statistics-random variable and probability theory-in studying the kinetic molecular theory. Namely, the behavior of the ideal gas, which depended on the probability density function, was conducted in the kinetic molecular theory. However, the focus of many researchers is controlling the volume of the ideal gas and comparing the relationship to the pressure, the temperature, and the number of the gas molecule by Boyle's law, Charles's law, Avogadro's law, respectively [1,19]. However, two of the significant factors that should be controlled in studying the kinetic molecular theory of the ideal gas are the velocity and kinetic energy of the molecule. Moreover, the statistical control chart for the kinetic molecular theory is a little employment. In particular, the studies of the relationship between the average run length of molecular velocity and kinetic energy with varied parameters, temperature, or mass of the molecule were hardly proposed.

Therefore, this research was conducted to study the EWMA control chart and its average run length for controlling the velocity and kinetic energy of an ideal gas. The numerical method for the evaluation of the integral equation representing average run length was carried out. The following section consists of the background and the method for derivation of EWMA control chart with one-sided upper control limit, probability theory for molecular gas, and computation of average run length for controlling velocity and kinetic energy of the ideal gas. The research also focused on evaluating the average run length of $\mathrm{N}$ molecules of the gas molecules in a system, assuming that each molecule is an identically independent distribution. In addition, the results, discussions, and conclusions are provided for this research.

\section{Materials and methods}

\section{Probability density function of molecular velocity}

Distribution of molecular velocity

In this section, the derivation of the formula of probability density function of molecular velocity in an ideal gas is proposed. Let $V$ be a random variable representing the velocity of molecular of an ideal gas. Let $f_{V}(v) d v$ be the probability density function of molecular velocity $V\left[\mathrm{~ms}^{-1}\right]$ between $v$ and $v+d v$. The expected number $N(v, v+d v)$ of molecules moving with velocity between $v$ and $v+d v$ is;

$$
N(v, v+d v)=N \int_{v}^{v+d v} f_{V}(v) d v
$$

where $N$ is dimensionless of the unit of $f_{V}(v)$ with seconds per meter. 
http://wjst.wu.ac.th

By the assumption, $\frac{d N}{N}$ is the fraction of molecules moving at velocity $v$ and $v+d v$, the number molecule with velocity between $v$ and $v+d v$ is the summation of the number of molecules in the sample times $f_{V}(v)$ times $d v$. This can be written in differential equation,

$d N(v, v+d v)=N f_{V}(v) d v$

Being carried out Eq. (1) by James Clerk Maxwell and Ludwig Boltzmann [20-23], the distribution function $f(v)$ of molecular velocity in an ideal gas at temperature $T[K]$ is called Maxwell-Boltzmann distribution, i.e.

$f_{V}(v)=\frac{4}{\sqrt{\pi}}\left(\frac{m}{2 K_{B} T}\right)^{3 / 2} v^{2} \exp \left\{\frac{m v^{2}}{2 K_{B} T}\right\} ; v>0$

where, $m[\mathrm{amu}]$ the mass of one gas molecule, $M[\mathrm{~g} / \mathrm{mol}]$ is the molar mass of the gas, which is $N_{A} m, N_{A}$ is the Avogadro constant $6.02214076 \times 10^{23} \mathrm{~mol}^{-1}, R$ is the gas constant which is $N_{A} K_{B}$, $K_{B}$ is the Boltzmann constant $1.3806 \times 10^{-23} \mathrm{~J} / \mathrm{K}$.

Expected Value and Variance of Molecular Velocity

The mean velocity $\langle V\rangle$ of molecule is the expected value of velocity distribution in Eq. (2), i.e.

$$
\begin{aligned}
<V>= & \int_{0}^{\infty} v f_{V}(v) d v \\
& =\frac{4}{\sqrt{\pi}}\left(\frac{m}{2 K_{B} T}\right)^{3 / 2} \int_{0}^{\infty} v^{3} \exp \left\{\frac{m v^{2}}{2 K_{B} T}\right\} d v
\end{aligned}
$$

Applying the technique of by part integral and improper integral,

$$
\begin{aligned}
<V> & =\lim _{b \rightarrow \infty} \frac{4}{\sqrt{\pi}}\left(\frac{m}{2 K_{B} T}\right)^{3 / 2} \int_{0}^{b} v^{3} \exp \left\{\frac{m v^{2}}{2 K_{B} T}\right\} d v \\
& \left.=\lim _{b \rightarrow \infty} \frac{4}{\sqrt{\pi}}\left(\frac{m}{2 K_{B} T}\right)^{3 / 2}\left[-\frac{\exp \left\{-\frac{m v^{2}}{2 K_{B} T}\right\}\left(1+\frac{m v^{2}}{2 K_{B} T}\right)}{2\left(\frac{m}{2 K_{B} T}\right)^{2}} v^{3}\right\}\right]_{v=0}^{v=b} \\
& =\sqrt{\frac{8 K_{B} T}{\pi m}} \\
& =\sqrt{\frac{8 R T}{\pi M}}
\end{aligned}
$$


http://wjst.wu.ac.th

The mean square velocity $\left\langle V^{2}\right\rangle$ of molecule is the second moment of the velocity distribution. Also, the root mean square velocity $\left(V_{r m s}\right)$ is the square root of the molecular velocity with median kinetic energy. The mean square velocity is,

$$
\begin{aligned}
<V^{2}>=\int_{0}^{\infty} v^{2} f_{V}(v) d v \\
=\frac{4}{\sqrt{\pi}}\left(\frac{m}{2 K_{B} T}\right)^{3 / 2} \int_{0}^{\infty} v^{4} \exp \left\{\frac{m v^{2}}{2 K_{B} T}\right\} d v
\end{aligned}
$$

Applying the technique of by part integral and improper integral,

$$
\begin{aligned}
<V^{2}>= & \lim _{t \rightarrow \infty} \frac{4}{\sqrt{\pi}}\left(\frac{m}{2 K_{B} T}\right)^{3 / 2} \int_{0}^{t} v^{4} \exp \left\{\frac{m v^{2}}{2 K_{B} T}\right\} d v \\
& =\lim _{t \rightarrow \infty} 4 \pi\left(\frac{m}{2 \pi K_{B} T}\right)^{3 / 2}\left[\frac{-v\left(3+\frac{m}{K_{B} T} v^{2}\right) \exp \left(-\frac{2 m}{K_{B} T} v^{2}\right)}{4\left(\frac{2 m}{K_{B} T}\right)^{2}}+\frac{3 \sqrt{\pi} \operatorname{erf}\left(\sqrt{\frac{2 m}{K_{B} T}} v\right)}{8\left(\frac{2 m}{K_{B} T}\right)^{5 / 2}}\right]_{v=0}^{v=t} \\
& =\frac{3 K_{B} T}{m} \\
& =\frac{3 R T}{M}
\end{aligned}
$$

The root mean square velocity of molecule is,

$$
V_{r m s}=\sqrt{\frac{3 K_{B} T}{m}}
$$

The variance of molecular velocity is,

$$
\begin{aligned}
\operatorname{Var}(V)= & <V^{2}>-(<V>)^{2} \\
& =\frac{R T}{m}\left(3-\frac{8}{\pi}\right)
\end{aligned}
$$

Computation of EWMA control chart and its average run length for molecular velocity and kinetic energy

Transformation of molecular velocity to molecular kinetic energy

Let $V$ be the random variable of molecular velocity with probability density function $f_{V}(v)$ and $E$ be the random variable of kinetic energy of molecule with probability density function $f_{E}(e)$, i.e. $E=\frac{1}{2} m V^{2}$. The transformation between two random variables, $E$ and $V$, was carried out. Namely, the transformation $T^{*}$ which is one to one function on real line is defined as; 


$$
E=T^{*}(V)=T^{*} \circ V=\frac{1}{2} m V^{2}
$$

where $\circ$ is a composite operator.

The inverse image of transformation of the singleton set $\{v\}$ is $T^{*-1}(\{v\})=T^{*-1}\{v\}$. Obviously, $T^{*}$ is monotonically increasing transformation. That is,

If for all $v_{1}, v_{2}$ such that $v_{1} \geq v_{2}$, then $T^{*}\left(v_{1}\right) \geq T^{*}\left(v_{2}\right)$. Thus, the cumulative distribution function $F_{E}$ for the molecule kinetic energy , $E=\frac{1}{2} m V^{2}$, is;

$$
\begin{aligned}
F_{E}(e)=\operatorname{Pr} & \{E \leq e\} \\
& =\operatorname{Pr}\left\{\frac{1}{2} m V^{2} \leq e\right\} \\
& =F_{V}\left(\sqrt{\frac{2 e}{m}}\right)
\end{aligned}
$$

Differentiating with respect to $e$, the probability density function of molecular kinetic energy is;

$$
\begin{aligned}
f_{E}(e)= & \frac{d}{d e} F_{V}\left(\sqrt{\frac{2 e}{m}}\right) \\
= & \frac{4 \pi \sqrt{2 e}}{m^{3 / 2}}\left(\frac{m}{2 \pi K_{B} T}\right)^{3 / 2} \exp \left\{-\frac{e}{K_{B} T}\right\} \\
= & \frac{2}{\sqrt{\pi}}\left(\frac{1}{K_{B} T}\right)^{3 / 2} e^{1 / 2} \exp \left\{-\frac{e}{K_{B} T}\right\} ; e>0
\end{aligned}
$$

Moreover, the expected value $\langle E\rangle$ and variance $\operatorname{Var}(E)$ of molecular kinetic energy is carried out as;

$$
<E>=\frac{3}{2} \frac{m R T}{M}
$$

The fourth moment of the molecular velocity is

$$
\begin{aligned}
<V^{4}>=\int_{0}^{\infty} v^{4} f_{V}(v) d v \\
\quad=4 \pi\left(\frac{m}{2 \pi K_{B} T}\right)^{3 / 2} \int_{0}^{\infty} v^{6} \exp \left\{-\frac{m v^{2}}{2 K_{B} T}\right\} d v
\end{aligned}
$$

Applying the technique of by part integral and improper integral, 


$$
\begin{aligned}
<V^{4}>= & 15\left(\frac{K_{B} N_{A} T}{m N_{A}}\right)^{5 / 2} \\
= & 15\left(\frac{R T}{M}\right)^{5 / 2}
\end{aligned}
$$

The variance of the square of molecular velocity is;

$$
\begin{aligned}
\operatorname{Var}\left(V^{2}\right)= & <V^{4}>-\left(<V^{2}>\right)^{2} \\
& =15\left(\frac{R T}{M}\right)^{5 / 2}-\left(\frac{3 R T}{M}\right)^{2}
\end{aligned}
$$

The variance of the molecular kinetic energy is;

$$
\begin{aligned}
\operatorname{Var}(E)=\frac{1}{4} & m^{2} \operatorname{Var}\left(V^{2}\right) \\
= & \frac{1}{4} m^{2}\left[15\left(\frac{R T}{M}\right)^{5 / 2}-\left(\frac{3 R T}{M}\right)^{2}\right]
\end{aligned}
$$

\section{EWMA control chart of molecular velocity and kinetic energy}

Let $v_{t}$ be independent and identically distributed (iid) random sample of molecular velocity from probability density function $f_{V}(v)$. Let $e_{t}$ be iid random sample of molecular kinetic energy from probability density function $f_{E}(e)$. EWMA statistic $z_{t}$ has widely been used for quality control. The formula of EWMA statistic with observations from the molecular velocity and molecular kinetic energy can iteratively be defined with starting value of given $z_{0}$ as;

$z_{t}=\lambda d_{t}+(1-\lambda) z_{t-1} ; t=1,2,3, \ldots$

where $d_{t}$ is iid random sample either for the molecular velocity with probability density function in Eq. (2) or for the molecular kinetic energy with probability density function in Eq. (8), i.e. $d_{t}=\left\{v_{t}\right\}$ or $\left\{e_{t}\right\}$.

Iteratively,

$z_{t}=\lambda \sum_{i=0}^{t-1}(1-\lambda)^{t} d_{t-i}+(1-\lambda)^{t} z_{0}$

The expected value $<z_{t}^{v}>$ and variance $\sigma_{z_{t}^{\nu}}^{2}$ of EWMA statistic of the observations which are sampled from probability density function of iid molecular velocity are carried out as;

$$
<z_{t}^{v}>=\sqrt{\frac{8 R T}{\pi M}}
$$




$$
\sigma_{z_{t}^{v}}^{2}=\frac{\lambda R T}{M(2-\lambda)}\left(3-\frac{8}{\pi}\right)\left[1-(1-\lambda)^{2 t}\right]
$$

The expected value $\left\langle z_{t}^{e}>\right.$ and variance $\sigma_{z_{t}^{e}}^{2}$ of EWMA statistic of the observations which are sampled from probability density function of iid molecular kinetic energy are carried out as;

$$
\begin{aligned}
& <z_{t}^{e}>=\frac{3}{2} \frac{m R T}{M} \\
& \sigma_{z_{i}^{e}}^{2}=\frac{m^{2} \lambda}{4(2-\lambda)}\left[15\left(\frac{R T}{M}\right)^{5 / 2}-\left(\frac{3 R T}{M}\right)^{2}\right]\left[1-(1-\lambda)^{2 t}\right]
\end{aligned}
$$

The EWMA control chart consists of 3 levels as follows:

1) Upper Control Limit (UCL) $=\left\langle z_{t}\right\rangle+\kappa \sqrt{\sigma_{z_{t}}^{2}}$

2) Center Line (CL) $=\left\langle z_{t}\right\rangle$

3) Lower Control Limit (LCL) $=\left\langle z_{t}\right\rangle-\kappa \sqrt{\sigma_{z_{t}}^{2}}$

where $\kappa$ is the width of the control limits.

\section{Average length for molecular velocity and molecular kinetic energy}

For Fredholm integral equation with the second kind, it can be written as;

$$
l(u)=h(u)+\gamma \int_{a}^{b} k(u, w) l(w) d w
$$

where $l(u)$ is an unknown function,

$$
k(u, w) \text { is a kernel function, }
$$

$\gamma$ is an eigenvalue of integral equation,

$a, b$ are a lower and an upper limit of integral sign.

This research, average run length or the solution of integral equation which stands for $l$ to measure the efficiency of EWMA control chart with upper one side based EWMA statistic is demonstrated in this section. If $d_{1}$ gives $z_{1}$ in control state, then $L C L<z_{1}<U C L$;

$$
\begin{aligned}
& \operatorname{Pr}\left\{\frac{L C L-(1-\lambda) z_{0}}{\lambda}<v_{1}<\frac{U C L-(1-\lambda) z_{0}}{\lambda}\right\} \\
& =\int_{\frac{L C L-(1-\lambda) z_{0}}{\lambda}}^{\frac{U C L-(1-\lambda) z_{0}}{\lambda}} f\left(d_{1}\right) d\left(d_{1}\right)
\end{aligned}
$$

By Champ and Rigdon [24] with initial point $z_{0}=u$, then; 
http://wjst.wu.ac.th

$$
l(u)=1+\int_{\frac{L C L-(1-\lambda) z_{0}}{\lambda}}^{\frac{U C L-(1-\lambda) z_{0}}{\lambda}} l[(1-\lambda) u+\lambda y] f(y) d(y)
$$

where $y$ is a setting of $d_{i}$.

Changing variable of integral,

$$
l(u)=1+\frac{1}{\lambda} \int_{L C L}^{U C L} l(w) f\left(\frac{w}{\lambda}-\frac{(1-\lambda)}{\lambda} u\right) d w
$$

which is compared with Fredholm integral equation with the second kind (18),

$$
h(u)=1, \gamma=\frac{1}{\lambda}, k(u, w)=f\left[\frac{w}{\lambda}-\frac{(1-\lambda)}{\lambda} u\right]
$$

For upper one sided control chart, average run length for controlling molecular velocity is;

$$
\begin{aligned}
l_{v}(u)= & 1+\frac{1}{\lambda} \int_{0}^{U C L} l_{v}(w) f_{V}\left(\frac{w}{\lambda}-\frac{(1-\lambda)}{\lambda} u\right) d w \\
& =1+A \int_{0}^{U C L} l_{v}(w)\left[\frac{w}{\lambda}-\frac{(1-\lambda)}{\lambda} u\right]^{2} \times \exp \left\{-\frac{m\left[\frac{w}{\lambda}-\frac{(1-\lambda)}{\lambda} u\right]^{2}}{2 K_{B} T}\right\} d w
\end{aligned}
$$

where $A=\frac{4}{\sqrt{\pi} \lambda}\left(\frac{m}{2 K_{B} T}\right)^{3 / 2}$.

For upper one sided control chart, average run length for controlling molecular kinetic energy is;

$$
\begin{aligned}
l_{e}(u)= & 1+\frac{1}{\lambda} \int_{0}^{U C L} l_{e}(w) f_{E}\left(\frac{w}{\lambda}-\frac{(1-\lambda)}{\lambda} u\right) d w \\
& =1+B \int_{0}^{U C L} l_{e}(w)\left[\frac{w}{\lambda}-\frac{(1-\lambda)}{\lambda} u\right]^{1 / 2} \times \exp \left\{-\frac{\left[\frac{w}{\lambda}-\frac{(1-\lambda)}{\lambda} u\right.}{K_{B} T}\right\} d w
\end{aligned}
$$

where $B=\frac{2}{\lambda \sqrt{\pi}}\left(\frac{1}{K_{B} T}\right)^{3 / 2}$. 
http://wjst.wu.ac.th

Numerical method of integral equation for average length for molecular velocity and molecular kinetic energy

Trapezoidal rule method [8] for integration is applied to solve the integral Eq. (20) - (21) for evaluating the average run length. This method was based on estimation the area under the curve with linear function of Trapezoidal. The partition of $N$ nodes for interval $[0, U C L]$ is constructed by equal length $h$ of each partition, i.e.

$h=\frac{U C L-0}{N} ; w_{i}=0+i h ; i=0,1,2, \ldots, N$

Therefore, the integral term $\int_{0}^{U C L} k(u, w) l(w) d w$ can be estimated as;

$\int_{0}^{U C L} k(u, w) l(w) d w=\frac{h}{2}\left[k\left(u, w_{0}\right) l\left(w_{0}\right)+k\left(u, w_{N}\right) l\left(w_{N}\right)\right]+h \sum_{i=1}^{N-1} k\left(u, w_{i}\right) l\left(w_{i}\right)$

Substituting Eq. (22) in to (19),

$l(u)=h(u)+\frac{h}{2}\left[k\left(u, w_{0}\right) l\left(w_{0}\right)+k\left(u, w_{N}\right) l\left(w_{N}\right)\right]+h \sum_{i=1}^{N-1} k\left(u, w_{i}\right) l\left(w_{i}\right)$.

Eq. (23) can be rewritten in a matrix notation for linear system of $N+1$ linear equations with $N+1$ unknowns as

$(I+K U) L=1$

$L=(I+K U)^{-1} 1$

where $1=[1,1, \ldots, 1]^{t}, L=\left[l_{i}\right]^{t}, K=\left[k_{i j}\right]_{j=1}^{N+1}, I$ is identity matrix, and $U=\operatorname{diag}\left(\frac{h}{2}, h, \ldots, h, \frac{h}{2}\right)$.

Eq. (24) is numerical solution of integral equation represented by average run length for molecular velocity and kinetic energy.

\section{Total error of trapezoidal rule for average run length}

Total error is all of the error in estimate the average run length. By the definition of integral in calculus,

$$
\begin{aligned}
I_{i} & =\int_{w_{i-1}}^{w_{i}} k(u, w) l(w) d w \\
& =K^{*}\left(u, w_{i}\right) L^{*}\left(w_{i}\right)-K^{*}\left(u, w_{i-1}\right) L^{*}\left(w_{i-1}\right)
\end{aligned}
$$

where $K^{*}(u, w) L^{*}(w)$ is an anti-derivative of $k(u, w) l(w)$.

With Taylor's series of $K^{*}(u, w) L^{*}(w)$ about $w_{i}$,

$$
K^{*}\left(u, w_{i-1}\right) L^{*}\left(w_{i-1}\right)
$$


$=K^{*}\left(u, w_{i}-h\right) L^{*}\left(w_{i}-h\right)$

$=K^{*}\left(u, w_{i}\right) L^{*}\left(w_{i}\right)-h \frac{\partial}{\partial w} K^{*}\left(u, w_{i}\right) L^{*}\left(w_{i}\right)+\frac{h^{2}}{2 !} \frac{\partial^{2}}{\partial w^{2}} K^{*}\left(u, w_{i}\right) L^{*}\left(w_{i}\right)$

$-\frac{h^{3}}{3 !} \frac{\partial^{3}}{\partial w^{2}} K^{*}\left(u, w_{i}\right) L^{*}\left(w_{i}\right)+\ldots$

$K^{*}\left(u, w_{i}\right) L^{*}\left(w_{i}\right)-K^{*}\left(u, w_{i-1}\right) L^{*}\left(w_{i-1}\right)$

$=h \frac{\partial}{\partial w} K^{*}\left(u, w_{i}\right) L^{*}\left(w_{i}\right)-\frac{h^{2}}{2 !} \frac{\partial^{2}}{\partial w^{2}} K^{*}\left(u, w_{i}\right) L^{*}\left(w_{i}\right)$

$+\frac{h^{3}}{3 !} \frac{\partial^{3}}{\partial w^{2}} K^{*}\left(u, w_{i}\right) L^{*}\left(w_{i}\right)-\ldots$

$=I_{i}$

Thus, $I_{i}=h k\left(u, w_{i}\right) l\left(w_{i}\right)-\frac{h^{2}}{2 !}\left[k\left(u, w_{i}\right) l\left(w_{i}\right)\right]^{\prime}+\frac{h^{3}}{3 !}\left[k\left(u, w_{i}\right) l\left(w_{i}\right)\right]^{\prime \prime}-\ldots$

By approximation of derivative of the integrand function, $\left[k\left(u, w_{i}\right) l\left(w_{i}\right)\right]^{\prime} \approx \frac{\left[k\left(u, w_{i}\right) l\left(w_{i}\right)\right]-\left[k\left(u, w_{i-1}\right) l\left(w_{i-1}\right)\right]}{h}+\frac{h}{2}\left[k\left(u, w_{i}\right) l\left(w_{i}\right)\right]^{\prime \prime}+.$.

Therefore,

$I_{i}=\frac{h^{2}}{2 !} \frac{\left[k\left(u, w_{i}\right) l\left(w_{i}\right)\right]-\left[k\left(u, w_{i-1}\right) l\left(w_{i-1}\right)\right]}{h}$

$-\frac{h^{3}}{12}\left[k\left(u, w_{i}\right) l\left(w_{i}\right)\right]^{\prime \prime}+\ldots$

$=C_{i}-\frac{h^{3}}{12}\left[k\left(u, w_{i}\right) l\left(w_{i}\right)\right]^{\prime \prime}+\ldots$.

Thus, the error term $E_{i}$ is

$E_{i}=I_{i}-C_{i}$

$=-\frac{h^{3}}{12}\left[k\left(u, w_{i}\right) l\left(w_{i}\right)\right]^{\prime \prime}+$ high-orderterms

where $C_{i}=\frac{h^{2}}{2 !} \frac{\left[k\left(u, w_{i}\right) l\left(w_{i}\right)\right]-\left[k\left(u, w_{i-1}\right) l\left(w_{i-1}\right)\right]}{h}$.

For $h \rightarrow 0, E_{i}=-\frac{h^{3}}{12}\left[k\left(u, w_{i}\right) l\left(w_{i}\right)\right]^{\prime \prime}$

If $\left|[k(u, w) l(w)]^{\prime \prime}\right| \leq M^{*}$, then $\left|E_{i}\right| \leq \frac{h^{3}}{12} M^{*}$.

For over the entire upper one sided control limit, the bound of total error $E_{T}$ is;

$\left|E_{T}\right| \leq \sum_{i=1}^{N} \frac{h^{3}}{12} M^{*}=N \frac{h^{3}}{12} M^{*}=(U C L) \frac{h^{3}}{12} M^{*}$. 
http://wjst.wu.ac.th

Therefore, $\left|E_{T}\right| \leq(U C L) \frac{h^{3}}{12} M^{*}$.

\section{Results and discussion}

In this section, the results from numerical method of integral equation representing average run length and analysis of its error are presented. As shown in the probability density, the function of the molecular velocity depends on the mass of a molecule and temperature, while the molecular kinetic energy depends only on the temperature. The variables for the experiment were determined by giving the mass of one gas molecule or the temperature.

\section{Probability density function of molecular velocity and kinetic energy of an ideal gas}

The example of an ideal gas is demonstrated by the assumption of setting the parameters as the following.

The main parameters are set as

$$
\begin{aligned}
& K_{B}=1.3806 \times 10^{\wedge}-23, \\
& N a=6.02214076 \times 10^{\wedge} 23, M=8, U C L=100, L C L=0 . \\
& m=1.328431254 \times 10^{\wedge}-23, \\
& m_{1}=1.328431254 \times 10^{\wedge}-23, \\
& m_{2}=2.328431254 \times 10^{\wedge}-23, \\
& m_{3}=3.328431254 \times 10^{\wedge}-23, \\
& m_{4}=4.328431254 \times 10^{\wedge}-23, \\
& m_{5}=5.328431254 \times 10^{\wedge}-23
\end{aligned}
$$

Figures 1 and 2 show the probability density function of molecular velocity for one molecule with $m$ and for mass $\left\{m_{1}, m_{2}, m_{3}, m_{4}, m_{5}\right\}$ of $\mathrm{N}=5$ molecules, respectively. Settings of the parameters are based on fixing the mass of one molecule and $\mathrm{N}=5$ molecules and varying temperature as $T=\{300,400,500,600,700\}$.

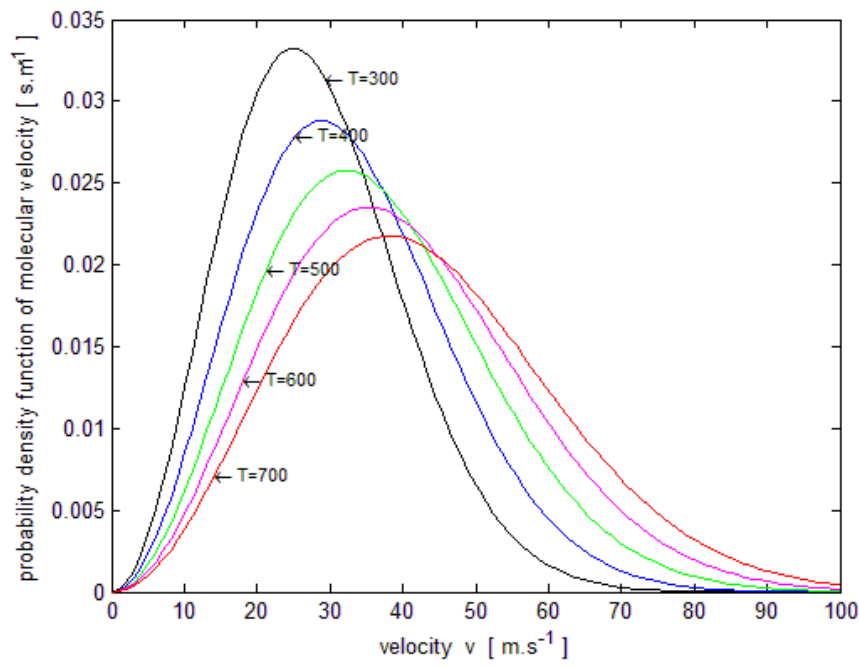

Figure 1 Probability density function of molecular velocity with varying temperature for one molecule. 
http://wjst.wu.ac.th

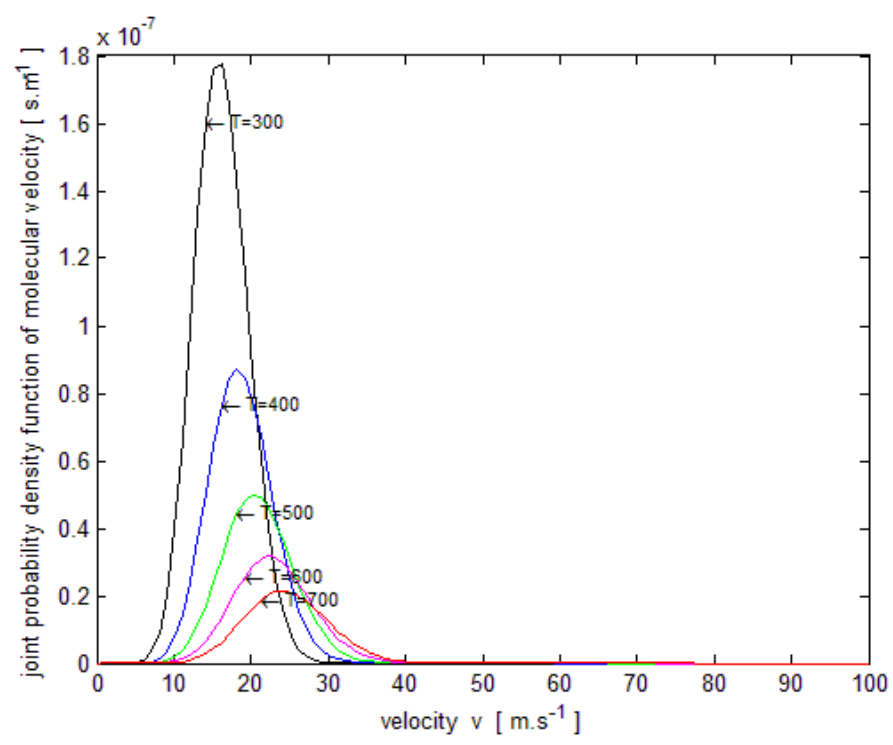

Figure 2 Probability density function of molecular velocity with varying temperature for $\mathrm{N}=5$ molecules.

Figures 3 and 4 show the probability density function of molecular velocity on fixing the temperature , $T=300$, with varying the mass of the one molecule $\{m, 2 m, 3 m, 4 m, 5 m\}$ and for mass $\left\{k m_{1}, k m_{2}, k m_{3}, k m_{4}, k m_{5}\right\} \quad k=1,2, \ldots, 5$ of $\mathrm{N}=5$ molecules, respectively.

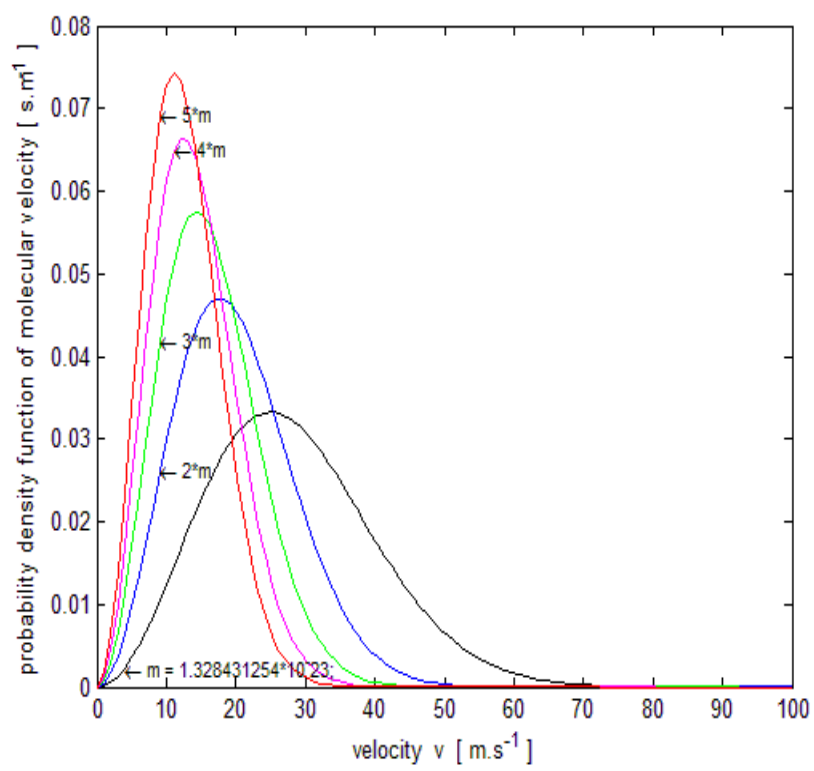

Figure 3 Probability density function of molecular velocity with varying the mass of one molecule. 
http://wjst.wu.ac.th

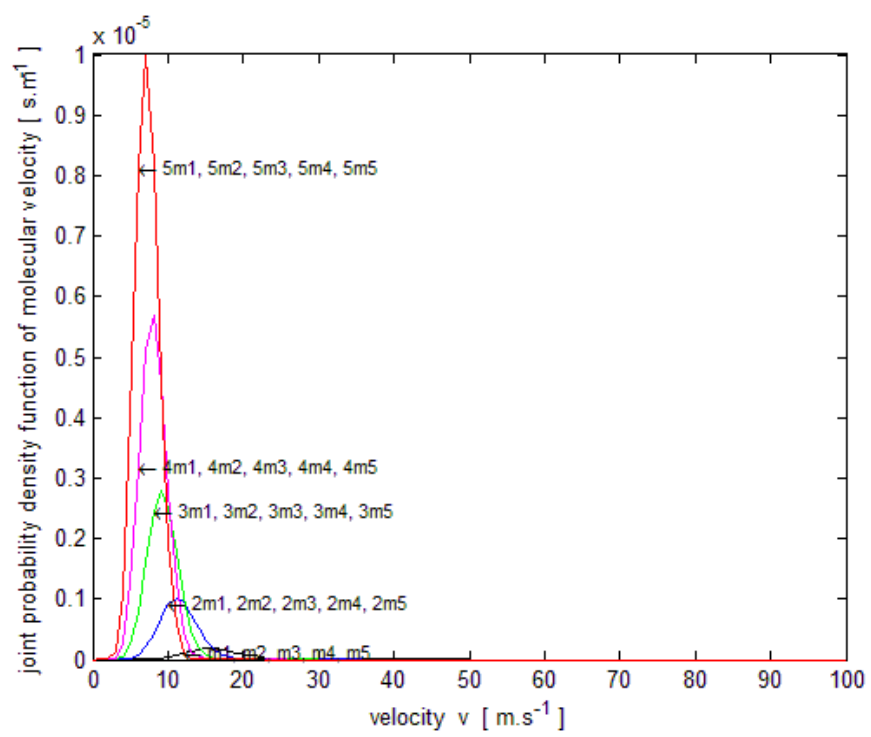

Figure 4 Probability density function of molecular velocity with varying the mass of $\mathrm{N}=5$ molecules.

Figures 5 and $\mathbf{6}$ show the probability density function of molecular kinetic energy for one molecule with $m$ and for mass $\left\{m_{1}, m_{2}, m_{3}, m_{4}, m_{5}\right\}$ of $\mathrm{N}=5$ molecules, respectively. Settings of the parameters are based on fixing the mass of one molecule and $\mathrm{N}=5$ molecules and varying temperature as $T=\{300,400,500,600,700\}$.

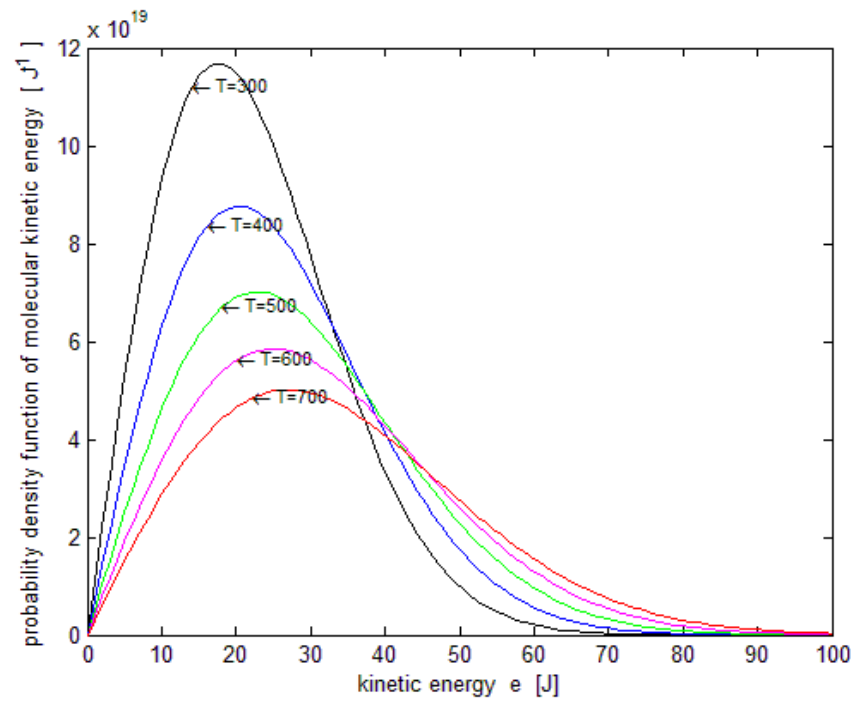

Figure 5 Probability density function of molecular kinetic energy with varying temperature for one molecule. 
http://wjst.wu.ac.th

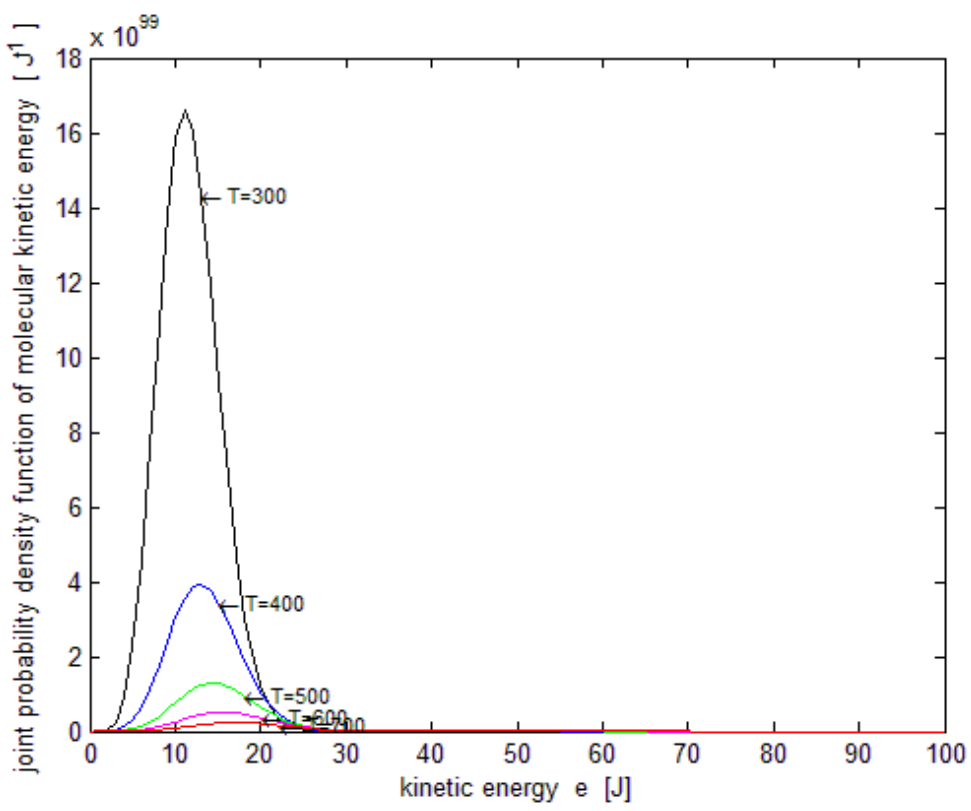

Figure 6 Probability density function of molecular kinetic energy with varying temperature for $\mathrm{N}=5$ molecules.

The results demonstrated from the probability density function are as follows:

In Figures 1 and 2, at the same mass of a molecule, when the temperature decreases, the probability density function shifts to lower and lower molecular velocity and vice versa. That is, the higher temperature makes the higher molecular velocity. In Figures 3 and 4, at the same temperature, when the mass of a molecule increases, the probability density function shifts to higher and higher velocity and vice versa. That is, the lower mass of a molecule makes the higher molecular velocity. In Figures 5 and $\mathbf{6}$, when the temperature increases, the probability density function shifts to higher and higher energy and vice versa. That is, the higher temperature makes the higher molecular kinetic energy.

\section{Average run length of molecular velocity and kinetic energy}

The assumptions of this experiment are as follows:

For average run length of molecular velocity, given temperature and varied mass of molecule were assigned. For average run length of molecular kinetic energy, given mass of a molecule and varied temperature were assigned. 
Table 1 Average run length of molecular velocity and kinetic energy for one molecule.

\begin{tabular}{|c|c|c|c|c|}
\hline \multirow{2}{*}{$\begin{array}{l}\text { Parameter } \\
(T)\end{array}$} & \multicolumn{2}{|c|}{$\begin{array}{l}\text { Average Run Length } \\
m=1.328431254 \times 10^{\wedge}-23\end{array}$} & \multirow{2}{*}{$\begin{array}{l}\text { Parameter } \\
(\mathrm{m})\end{array}$} & \multirow{2}{*}{$\begin{array}{l}\text { Average Run Length } \\
T=300\end{array}$} \\
\hline & Molecular Velocity & Molecular Kinetic Energy & & \\
\hline 300 & $\begin{array}{l}370 \\
\left(A R L_{0}\right)\end{array}$ & $\begin{array}{l}370 \\
\left(A R L_{0}\right)\end{array}$ & $m=1.328431254 \times 10^{-23}$ & $\begin{array}{l}370 \\
\left(A R L_{0}\right)\end{array}$ \\
\hline 400 & $366.30274^{\left(A R L_{1}\right)}$ & $\begin{array}{l}309.43765 \\
\left(A R L_{1}\right)\end{array}$ & $2 m$ & $\begin{array}{l}346.28428 \\
\left(A R L_{1}\right)\end{array}$ \\
\hline 500 & $359.69551\left(A R L_{1}\right)$ & $\begin{array}{l}193.97613 \\
\left(A R L_{1}\right)\end{array}$ & $3 m$ & $\begin{array}{l}276.85208 \\
\left(A R L_{1}\right)\end{array}$ \\
\hline 600 & $351.85221^{\left(A R L_{1}\right)}$ & $\begin{array}{l}176.58856 \\
\left(A R L_{1}\right)\end{array}$ & $4 m$ & $\begin{array}{l}171.82999 \\
\left(A R L_{1}\right)\end{array}$ \\
\hline 700 & $343.40859^{\left(A R L_{1}\right)}$ & $\begin{array}{l}152.135389 \\
\left(A R L_{1}\right)\end{array}$ & $5 m$ & $\begin{array}{l}79.63196 \\
\left(A R L_{1}\right)\end{array}$ \\
\hline 800 & $334.67125\left(A R L_{1}\right)$ & $\begin{array}{l}150.95026 \\
\left(A R L_{1}\right)\end{array}$ & $6 m$ & $\begin{array}{l}31.20898 \\
\left(A R L_{1}\right)\end{array}$ \\
\hline 900 & $\begin{array}{l}325.82837 \\
\left(A R L_{1}\right)\end{array}$ & $\begin{array}{l}148.06635 \\
\left(A R L_{1}\right)\end{array}$ & $7 m$ & $\begin{array}{l}12.97953 \\
\left(A R L_{1}\right)\end{array}$ \\
\hline 1,000 & $316.98928\left(A R L_{1}\right)$ & $\begin{array}{l}147.87615 \\
\left(A R L_{1}\right)\end{array}$ & $8 m$ & $\begin{array}{l}7.15292 \\
\left(A R L_{1}\right)\end{array}$ \\
\hline
\end{tabular}

Table 1 gives information about the average run length of molecular velocity and molecular kinetic energy of a gas molecule. There is a decrease in the average run length of molecular velocity when the temperature increases in the given molecular mass. Likewise, the average run length of molecular kinetic energy decreases when temperature increases with varied increasing temperatures. The average run length of molecular velocity decreases when the mass of the molecule increases with given temperature. However, the average run length of molecular kinetic energy does not depend on the molecule's mass.

Table 2 Average run length of molecular velocity and kinetic energy for $\mathrm{N}=5$ molecules.

\begin{tabular}{|c|c|c|c|c|}
\hline \multirow{2}{*}{$\begin{array}{l}\text { Parameter } \\
(T)\end{array}$} & \multicolumn{2}{|l|}{$\begin{array}{c}\text { Average Run Length } \\
m_{1}, m_{2}, m_{3}, m_{4}, m_{5}\end{array}$} & \multirow[t]{2}{*}{$\begin{array}{l}\text { Parameter } \\
(\mathrm{m})\end{array}$} & \multirow{2}{*}{$\begin{array}{l}\text { Average Run Length } \\
T=300 \\
\text { Molecular Velocity }\end{array}$} \\
\hline & Molecular Velocity & Molecular Kinetic Energy & & \\
\hline$T_{1}=300$ & $\begin{array}{l}370 \\
\left(A R L_{0}\right)\end{array}$ & $\begin{array}{l}370 \\
\left(A R L_{0}\right)\end{array}$ & $m_{1}, m_{2}, m_{3}, m_{4}, m_{5}$ & $\begin{array}{l}370 \\
\left(A R L_{0}\right)\end{array}$ \\
\hline$T_{2}=400$ & $367.16274\left(A R L_{1}\right)$ & $354.71156\left(A R L_{1}\right)$ & $2 m_{1}, 2 m_{2}, 2 m_{3}, 2 m_{4}, 2 m_{5}$ & $\begin{array}{l}345.83321 \\
\left(A R L_{1}\right)\end{array}$ \\
\hline$T_{3}=500$ & $365.19354\left(A R L_{1}\right)$ & $305.14327\left(A R L_{1}\right)$ & $3 m_{1}, 3 m_{2}, 3 m_{3}, 3 m_{4}, 3 m_{5}$ & $\begin{array}{l}327.63099 \\
\left(A R L_{1}\right)\end{array}$ \\
\hline
\end{tabular}




\begin{tabular}{lllll}
\hline $\begin{array}{l}\text { Parameter } \\
(T)\end{array}$ & $\begin{array}{l}\text { Average Run Length } \\
m_{1}, m_{2}, m_{3}, m_{4}, m_{5}\end{array}$ & $\begin{array}{l}\text { Parameter } \\
(\mathrm{m})\end{array}$ & \multicolumn{1}{c}{$\begin{array}{l}\text { Average Run Length } \\
T=300\end{array}$} \\
\cline { 2 - 5 } & Molecular Velocity & Molecular Kinetic Energy & & \multicolumn{1}{c}{ Molecular Velocity } \\
\hline$T_{4}=600$ & $364.68180\left(A R L_{1}\right)$ & $235.07539\left(A R L_{1}\right)$ & $4 m_{1}, 4 m_{2}, 4 m_{3}, 4 m_{4}, 4 m_{5}$ & $\begin{array}{l}312.38195 \\
\left(A R L_{1}\right)\end{array}$ \\
\hline$T_{5}=700$ & $364.51958\left(A R L_{1}\right)$ & $221.58643\left(A R L_{1}\right)$ & $5 m_{1}, 5 m_{2}, 5 m_{3}, 5 m_{4}, 5 m_{5}$ & 254.53605 \\
& & & & $\left(A R L_{1}\right)$ \\
\hline$T_{6}=800$ & $364.18934\left(A R L_{1}\right)$ & $128.88655\left(A R L_{1}\right)$ & $6 m_{1}, 6 m_{2}, 6 m_{3}, 6 m_{4}, 6 m_{5}$ & 238.58292 \\
& & & & $\left(A R L_{1}\right)$ \\
\hline$T_{7}=900$ & $361.65251\left(A R L_{1}\right)$ & $119.77965\left(A R L_{1}\right)$ & $7 m_{1}, 7 m_{2}, 7 m_{3}, 7 m_{4}, 7 m_{5}$ & 150.01113 \\
& & & $8 m_{1}, 8 m_{2}, 8 m_{3}, 8 m_{4} 8 m_{5}$ & 96.685519 \\
\hline$T_{7}=1,000$ & $344.44424\left(A R L_{1}\right)$ & $118.90893\left(A R L_{1}\right)$ & & $\left(A R L_{1}\right)$ \\
\hline
\end{tabular}

Table 2 gives information about average run length of molecular velocity and molecular kinetic energy of $\mathrm{N}=5$ gas molecules. There is a decrease in average run length of molecular velocity when the temperature increases in a given molecular mass. Likewise, the average run length of molecular kinetic energy decreases when the temperature increases with varied increasing temperatures. Meanwhile, the average run length of the molecular velocity decreases when the mass of molecule increases with given temperatures. However, the average run length of molecular kinetic energy does not depend on the mass of molecule.

\section{Conclusions}

This research evaluates the average run length of molecular velocity and kinetic energy of a gas molecule and $\mathrm{N}$ molecules. The EWMA control chart is employed to construct the formula of average run length, which is the integral equation solution. Then, the numerical method for approximation of the solution of the integral equation is based on the Trapezoidal rule. For both cases of one molecule and $\mathrm{N}$ molecules, the results show an increase in molecular velocity and kinetic energy when the temperature increases with given the mass of the molecule. That is, the molecule gets more the temperature, then the molecule will move faster. This makes the molecular kinetic energy increases as well because the molecular kinetic energy has a relationship with squared molecular velocity. Furthermore, at the same temperature, the molecular velocity increases when the mass of the molecule decreases. It implies that the molecule moves faster when the molecular weight is lighter. To sum up, the relationship between the average run length of molecular velocity or kinetic energy with varying parameters: temperature and mass of the molecule is conducted. The results show that the average run length has decreased when there is an increase in temperature with a given mass of the molecule. Similarly, there is a decrease in average run length when the mass of the molecule increases with the given temperature of the molecule. Namely, the parameters: temperature and mass of the molecule affect the average run length of a gas molecule's molecular velocity and kinetic energy. Furthermore, the other control charts are applied to this field with autocorrelation of the data to detect the change points on a control chart. This alternative should be conducted for future works and novel researches.

\section{Acknowledgements}

The research was funded by King Mongkut's University of Technology North Bangkok Contract no. KMUTNB-60-GOV-18. 


\section{References}

[1] IR Levine. Physical Chemistry. $6^{\text {th }}$ eds. Tata McGraw-Hill, New Delhi, 2008.

[2] J Rodenburg, S Paliwal, MD Jager, PG Bolhuis, M Dijkstra and RV Roij. Ratchet-induced variations in bulk states of an active ideal gas. J. Chem. Phys. 2018; 149, 1-12.

[3] H Hernandez. Calculation of Molecular Fluxes and Equivalent Pressure in Ideal Gases. For. Chem. Res. 2019; 3, 1-31.

[4] FK Reinhart. Ideal gas Law and the Greenhouse Effect. J. Ear. Sci. Clim. Cha. 2018; 9, 1-2.

[5] RI Holmes. Molar mass version of the ideal gas law points to a very low climate sensitivity. Ear. Sci. 2017; 6, 157-63.

[6] H Varaee and MR Ghasemi. Engineering optimization based on ideal gas molecular movement algorithm. Eng. Comput. 2017; 33, 71-93.

[7] J Huang, F Ching and J Cheng. The study on the Boltzmann distribution of ideal gas under gravitational field by computer simulation. Adv. Mate. Res. 2014; 939, 584-91.

[8] A Kharab and RB Guenther. An Introduction to Numerical methods A MATLAB Approach. $3^{\text {rd }}$ eds. CRC Press, USA, 2012.

[9] RR Nigmatullin and P Agarwal. Direct evaluation of the desired correlations: Verification on real data. Physica A Stat. Mec. Its Appl. 2019; 534, 121558.

[10] GM Tuncay and P Agarwal. Use of quantum differential equations in sonic processes. App. Math. Nonlin. Sci. 2020; 6, 1-8.

[11] P Agarwal, M Attary, M Maghasedi and P Kumam. Solving higher-order boundary and initial Value problems via Chebyshev-Spectral method: Application in elastic foundation. Symmetry 2020; 12, 987.

[12] P Agarwal, U Baltaeva and A Yolqin. Solvability of the boundary-value problem for a linear loaded integro-differential equation in an infinite three-dimensional domain. Chaos Solitons Fractals 2020; 140, 110108

[13] Y Areepong and AA Novikov. Martingale approach to EWMA control chart for changes in exponential distribution. J. Qual. Meas. Anal. 2008; 4, 197-203.

[14] K Petcharat, Y Areepong, S Sukparungsee and G Mititelu. Fitting pareto distribution with hyperexponential to evaluate the ARL for CUSUM chart. Int. J. Pur. Appl. Math. 2012; 77, 233-44.

[15] R Taboran, S Sukparungsee and Y Areepong. A new nonparametric Tukey MA-EWMA control charts for detecting mean shifts. IEEE Access 2020; 8, 207249-59.

[16] G Mititelu, Y Areepong, S Sukparungsee and A Novikov. Explicit analytical solutions for the average run length of CUSUM and EWMA charts. Eas. Wes. J. Math. 2010; SI, 253-65.

[17] R Sunthornwat, Y Areepong and S Sukparungsee. Average run length of the long-memory autoregressive fractionally integrated moving average process of the exponential weighted moving average control chart. Cog. Math. 2017; 4, 1-11.

[18] Ugaza and Sáncheza. Adaptive EWMA control charts with a time varying smoothing parameter. Stat. Econ. 2015; 15, 1-31.

[19] P Atkins and JD Paula. Atkin's Physical Chemistry. $11^{\text {th }}$ eds. Oxford University Press, UK, 2018.

[20] JRH Tame. Maxwell and Boltzmann In: Approaches to Entropy. Springer, Singapore, 2019.

[21] JS Rowlinson. The Maxwell-Boltzmann distribution. Mole. Phys. 2005; 103, 2821-828.

[22] RC Dunbar. Deriving the Maxwell distribution. J. Chem. Educ. 1982; 59, 22-3.

[23] GD Peckham and IJ McNaught. Applications of the Maxwell-Boltzmann distribution. J. Chem. Educ. 1992; 69, 554-58.

[24] C Champ and S Rigdon. A comparison of the Markov chain and the integral equation approaches for evaluating the run length distribution of quality control charts. Commun. Stat. Simu. Comput. 1991; 20, 191-203. 